\title{
On the cooling-schedule dependence of the dynamics of mean-field glasses
}

\author{
Andrea Montanari \\ Laboratoire de Physique Théorique de l'Ecole Normale Supérieure*, \\ 24, rue Lhomond, 75231 Paris CEDEX 05, France \\ Federico Ricci-Tersenghi \\ Dipartimento di Fisica, INFM (UdR Roma I and SMC center) \\ Università di Roma "La Sapienza", \\ P. Aldo Moro 2, I-00185 Roma, Italy
}

\begin{abstract}
The low temperature phase of discontinuous mean-field spin glasses is characterized by the appearance of an exponential number of metastable states. Which ones among these states dominate the out-of-equilibrium dynamics of these systems?

In order to answer this question, we compare high-precision numerical simulations of a diluted $p$-spin model with a cavity computation of the threshold energy. Our main conclusion is that the aging dynamics is dominated by different layers of metastable states depending on the cooling schedule. In order to perform our analysis, we present a method for computing the marginality condition of diluted spin glasses at non-zero temperature.
\end{abstract}

PACS numbers: 75.50.Lk,64.70.Pf

\footnotetext{
* UMR 8549, Unité Mixte de Recherche du Centre National de la Recherche Scientifique et de l' Ecole Normale Supérieure.
} 


\section{INTRODUCTION AND RESULTS}

Upon cooling, glass-forming liquids show a dramatic slowing-down of their relaxational dynamics. In mean-field models, this phenomenon is caricatured by a weak ergodicity breaking phase transition (the so called "dynamic phase transition") which occurs at some critical temperature $T_{\mathrm{d}}$ [1, 2]. Surprisingly enough, this phenomenon could be relevant in understanding the behavior of local search algorithm for hard computational problems [3, 4]. The time-complexity of these algorithms suddenly explodes when some macroscopic parameter describing the instances crosses a critical value.

Despite their apparent simplicity, the dynamics of mean-field models is still poorly understood in many aspects. Consider, for instance, the following couple of Gedanken experiments. In the first one, the system is at infinite temperature at time $t=0: T(0)=\infty$, and is suddenly quenched below the dynamic transition: $T(t)=T<T_{\mathrm{d}}$ for $t>0$. At some time $t_{\mathrm{f}}$ we measure the observable $\mathcal{O}$ and the experiment is finished. We are interested in the asymptotic behavior:

$$
\langle\mathcal{O}\rangle_{\text {quench }} \equiv \lim _{t_{\mathrm{f}} \rightarrow \infty}\left\langle\mathcal{O}\left(t_{\mathrm{f}}\right)\right\rangle_{T}
$$

In the second experiment we choose a cooling schedule, i.e. a smooth function $T_{\text {sch }}(\tau)$, $0 \leq \tau \leq 1$, with $T_{\text {sch }}(0)=\infty$ and $T_{\text {sch }}(1)=T$. We start from $T(0)=\infty$ and slowly cool down the system, keeping it at temperature $T(t)=T_{\mathrm{sch}}\left(t / t_{\mathrm{f}}\right)$ for $0 \leq t \leq t_{\mathrm{f}}$. Then we measure the same observable $\mathcal{O}$ and consider the limit

$$
\langle\mathcal{O}\rangle_{\text {cool }} \equiv \lim _{t_{\mathrm{f}} \rightarrow \infty}\left\langle\mathcal{O}\left(t_{\mathrm{f}}\right)\right\rangle_{T_{\text {sch }}\left(t / t_{\mathrm{f}}\right)}
$$

In this paper we address the question:

$$
\langle\mathcal{O}\rangle_{\text {quench }} \stackrel{?}{=}\langle\mathcal{O}\rangle_{\text {cool }}
$$

Notice that both in Eqs. (11) and (2), the thermodynamic limit is assumed to be taken before the long-time limit. In the opposite case the answer would be trivially positive.

It turns out that the two quantities are in general different: $\langle\mathcal{O}\rangle_{\text {quench }} \neq\langle\mathcal{O}\rangle_{\text {cool }}$. In the following we shall focus on the internal energy, $\mathcal{O}=\mathcal{H}(\sigma)$ and we will show that in this case $\langle\mathcal{H}(\sigma)\rangle_{\text {quench }}>\langle\mathcal{H}(\sigma)\rangle_{\text {cool }}$ strictly. This result is quite surprising. The bulk of analytical

results in this field comes, in fact, from the solution of the fully connected $p$-spin spherical model [5, 6, 7]. In this case $\langle\mathcal{O}\rangle_{\text {quench }}=\langle\mathcal{O}\rangle_{\text {cool }}$. 
Like other properties of the out-of-equilibrium dynamics of mean-field models, the result $\langle\mathcal{O}\rangle_{\text {quench }} \neq\langle\mathcal{O}\rangle_{\text {cool }}$ can be interpreted in connection with the structure of metastable states. It is worth recalling this connection here. Below $T_{\mathrm{d}}$ the Boltzmann measure splits into an exponential number of metastable states. Formally [23]

$$
\langle\mathcal{O}\rangle_{\mathrm{eq}} \approx \sum_{\alpha} w_{\alpha}\langle\mathcal{O}\rangle_{\alpha} .
$$

Such a splitting has been proven rigorously for some completely connected $p$-spin models [8]. Two different states have different values of the extensive observables (energy, magnetization, etc). One can therefore construct constrained Boltzmann measures using only those states which have a definite value of one of these observables. For instance one can constrain on the energy

$$
\langle\mathcal{O}\rangle_{e} \propto \sum_{\alpha:\langle\mathcal{H}\rangle_{\alpha} \approx N e} w_{\alpha}\langle\mathcal{O}\rangle_{\alpha} .
$$

It is a simple property of mean field models that purely relaxation dynamics does not necessarily converge to (Boltzmann) equilibrium. Consider for instance a Curie Weiss model at low temperature in a small positive magnetic field. If the dynamics is initiated from a (sufficiently) negative magnetization configuration, it will converge to a negative magnetization state unless one waits a time exponential in the size of the system [9].

Unlike in the Curie Weiss model, in glassy systems it is difficult to select a particular metastable state by switching on a field in the appropriate direction. In general, the appropriate out-of-equilibrium measure will rather resemble a mixture of metastable states. A fascinating hypothesis on mean-field spin glasses is that relaxation dynamics converges indeed (as long as one-time observables are concerned) to a constrained measure of the form (5). If one accepts this hypothesis, the open question is: which of the many possible constrained measures does the dynamics converge to? Our results imply that the answer depends upon the path along which the system is driven into its low-temperature phase. Viceversa, this path can be tailored in such a way to select different constrained measures.

Figure 1 sketches the mechanism for selecting metastable states at different energy densities by changing the cooling schedule. The evolution of the energy density and its asymptotic value are traced. In the inset we show the complexity (i.e. the logarithm of the number of metastable states) at the final temperature. It turns out that cooling slowly the system through the dynamic transition one is able to avoid the higher energy (and most numerous) 

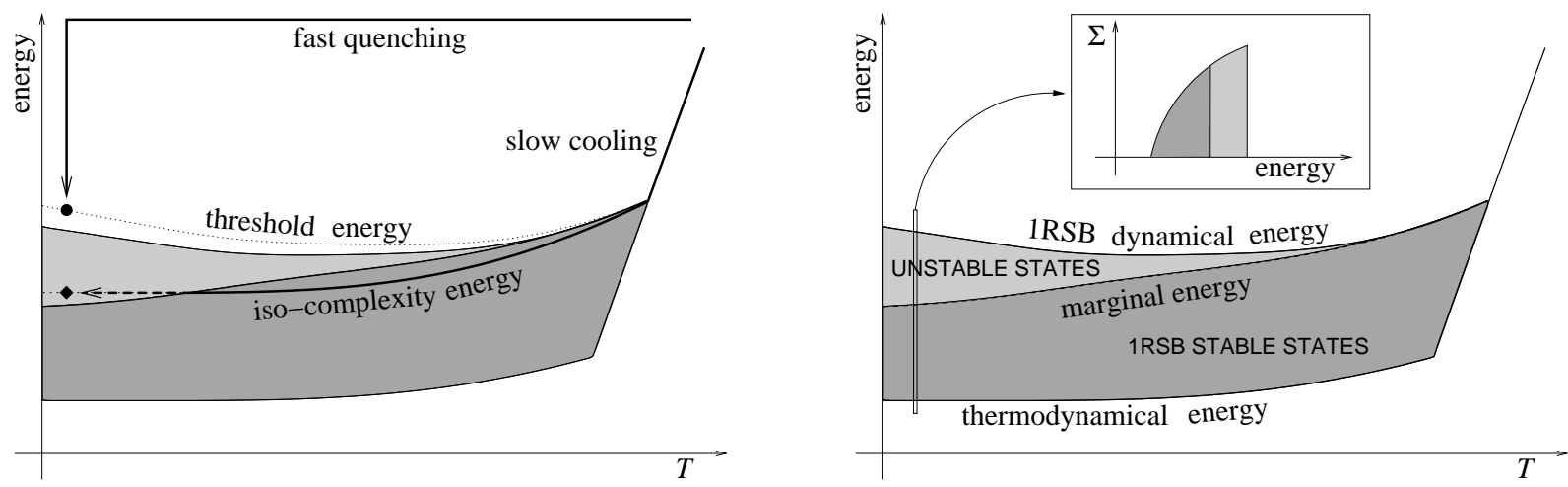

FIG. 1: A pictorial view of the temperature evolution of metastable states in a discontinuous mean-field model, without temperature chaos. The gray areas correspond to those regions in the temperature-energy plane, such that an exponential number of metastable states is present, within a 1RSB calculation. Dark gray corresponds to states which are stable with respect to FRSB, while light gray corresponds to unstable states. On the left we depict the trajectory of a quench and a cooling protocols. On the right (inset) we plot the 1RSB complexity (i.e. the entropy of metastable states) at a particular temperature. By 'threshold states', we mean here the highest energy states such that the complexity is strictly positive. In general FRSB must be used to describe these states, as well as to calculate the iso-complexity energy in the light gray region (dashed line).

metastable states. This is not the case after a rapid quench. We also show the marginal energy density which correspond to the appearance of a full hierarchy of 'nested' decompositions of the form (4). In spin glass jargon, this corresponds to full replica symmetry breaking (FRSB). Let us notice that these sketches, as well as the examples in the following, refer to cases in which a thermodynamic FRSB transition does not exist (in other words the marginal energy line does not cross the thermodynamical energy line). This is not, however, a fundamental restriction of the theory.

Already Crisanti, Horner and Sommers 6] noticed that the asymptotic value of one-time observables depends upon the cooling schedule for the spherical $p$-spin model in non-zero magnetic field. This phenomenon was further investigated in a non-homogeneous spherical model by Barrat, Franz and Parisi [10, 11]. The crucial point is that the $p$-spin spherical model is an exceptional case as far as the structure of high-lying metastable states is considered.

Although several conclusions of [1]] agree with our findings, they relied on some uncontrolled assumptions and interpretations. The generality of their results wasn't fully 
appreciated. In this paper we revisit the same problem in a context [12], where numerical simulations can be carefully compared with analytical results, and assumptions can be checked. Moreover, the model considered is a close (although simpler) relative of a large family of hard optimization problems.

Our conclusions are based on an extensive numerical study of a diluted Ising spin glass with $p$-spin interactions. The dynamics of these models can be simulated with a smaller computational effort with respect to their fully-connected counterparts. Moreover, the analysis of their static properties has being rapidly developing in the last few years. This paper contains one further step in this direction. Developing the ideas of Ref. [12], we show how to compute the marginality condition of one-step replica-symmetry breaking (1RSB) solutions for diluted models at non-zero temperature.

The paper is organized as follows. In Section [I] we define the family of models to be investigated and give some analytical results concerning the metastable states of these models. Moreover we explain how the marginality condition is computed. In Sec. III we present our numerical simulations and compare them with the static calculations mentioned above. A few comments on these results are presented in Sec. IV In Appendix A we collect some explicit formulae for the model of Sec. II] Finally in the App. B. we show that, in the large connectivity limit, our computation of the marginality condition coincides with the usual replicon result [13].

\section{THE MODEL AND ITS MARGINALITY CONDITION}

We shall consider the family of diluted spin glasses defined by the Hamiltonian

$$
\mathcal{H}(\sigma)=-\sum_{\left(i_{1} \ldots i_{p}\right) \in \mathcal{G}} J_{i_{1} \ldots i_{p}} \sigma_{i_{1}} \ldots \sigma_{i_{p}}
$$

where $\mathcal{G}$ is the hypergraph of interactions, i.e. a set of $M$ among the $\left(\begin{array}{l}N \\ p\end{array}\right)$ possible $p$-uples (with $p \geq 3$ ) of the $N$ spins. We shall consider hypergraphs $\mathcal{G}$ with fixed connectivity: each spin is supposed to participate to $c=l+1$ interaction terms (hereafter $c \geq 3$ ). The graph $\mathcal{G}$ is drawn randomly, with uniform probability, among all such hypergraphs (i.e. among all the hypergraphs on $N$ vertices with connectivity $c$, and $p$-vertices edges). Finally we shall assume the couplings $J_{i_{1} \ldots i_{p}}$ to take the values \pm 1 with equal probability.

This model was already studied in Refs. [14, 15] within a 1RSB approximation. As the 


\begin{tabular}{|c|c|c|c|c||c|c||c|}
\hline$c$ & $T_{\mathrm{s}}$ & $T_{\mathrm{d}}$ & $\Sigma_{\mathrm{d}}\left(T_{\mathrm{d}}\right)$ & $e_{\mathrm{d}}\left(T_{\mathrm{d}}\right)$ & $T_{\max }$ & $\Delta T$ & $T_{\text {quench }}$ \\
\hline \hline 4 & $0.655(5)$ & $0.757(5)$ & $0.0540(10)$ & $-1.157(2)$ & 1.0 & 0.01 & $0.4,0.5,0.6$ \\
5 & $0.849(5)$ & $0.936(5)$ & $0.0445(10)$ & $-1.314(2)$ & 1.5 & 0.01 & $0.6,0.7,0.8$ \\
8 & $1.25(1)$ & $1.345(5)$ & $0.0335(10)$ & $-1.683(5)$ & 2.0 & 0.02 & $0.8,1.0,1.2$ \\
\hline \hline
\end{tabular}

TABLE I: Critical temperatures for the static $\left(T_{\mathrm{s}}\right)$ and dynamic $\left(T_{\mathrm{d}}\right)$ transitions of the model (6) as obtained from a population dynamics solution of the 1RSB equations. We also report the complexity $\left(\Sigma_{\mathrm{d}}\right)$ and energy $\left(e_{\mathrm{d}}\right)$ of threshold states at the dynamic transition. Quoted errors include both statistical and systematic uncertainties. The two central columns refer to parameter values used in the numerical cooling experiments, while the last column contains the temperatures used for the quenching experiments.

temperature is lowered it undergoes a dynamic phase transition at $T_{\mathrm{d}}>0$ and, if $c>p$, a static phase transition at $T_{\mathrm{s}}$, with $0<T_{\mathrm{s}}<T_{\mathrm{d}}$. For $l \geq l_{\mathrm{G}}(p)$ a second static phase transition takes place at some temperature $T_{\mathrm{G}}$ : the $T<T_{\mathrm{G}}$ phase is characterized by full replica-symmetry breaking. In Ref. [12] we studied the $T=0$ limit and found $l_{\mathrm{G}}(3)=10$. In Tab. \we report the values of $T_{\mathrm{s}}$ and $T_{\mathrm{d}}$ for $p=3$ and a few values of $l<10$.

At a fixed temperature $T=1 / \beta<T_{\mathrm{d}}$, the Gibbs measure decomposes over an exponential number of pure (metastable) states. The number of states at a given free energy density $f$ is given, at the leading exponential order, by $\mathcal{N}_{\beta}(f) \sim \exp \left\{N \Sigma_{\beta}(f)\right\}$. In 1 RSB approximation, the complexity $\Sigma_{\beta}(f)$ is obtained [16] as the Legendre transform of the $m$ replicas free energy $m \phi(m, \beta)$ with respect to the parameter $m$ :

$$
\Sigma_{\beta}(f)=m \beta f-\left.m \phi(m, \beta)\right|_{\beta f=\partial_{m}[m \phi]}
$$

The parameter $m$ can be varied in the range $m_{\mathrm{d}}(T)<m<m_{\mathrm{s}}(T)$. This corresponds to selecting states of free-energy densities $f_{\mathrm{d}}(T)>f>f_{\mathrm{s}}(T)$. Any other observable can be computed over metastable states of a given free energy, by properly tuning $m$. The internal energy, for instance, decreases from $e_{\mathrm{d}}(T)$ to $e_{\mathrm{s}}(T)$, as $f$ varies from $f_{\mathrm{d}}(T)$ to $f_{\mathrm{s}}(T)$. In Tab. II we report the curves $e_{\mathrm{S}}(T)$ and $e_{\mathrm{d}}(T)$ for $p=3$ and a few values of $l$, see also Figs. 2, 3. The 1RSB saddle point equations were solved using the population dynamics algorithm of Ref. [17].

As shown in Ref. [12], high-energy metastable states are, quite generically, unstable towards FRSB. Here we want to compute the stability threshold $m_{\mathrm{G}}(T)$ and the corresponding 
internal energy $e_{\mathrm{G}}(T)$ at finite temperature $T$. We proceed by determining the instability toward two-steps replica symmetry breaking (2RSB), which in turn is expected to imply the instability towards FRSB. We shall present the method in a general setting and give some numerical results, cf. Tab. W. We refer to App. $\mathrm{A}$ for explicit formulae in the case of the Hamiltonian (6).

Let $\mathcal{S}$ be the space of normalized measures $\rho(x)$ over the real numbers. The $2 \mathrm{RSB}$ order parameter for the model ([6) is a probability distribution $Q[\rho]$ over $\mathcal{S}$. This is slightly simpler than the most general 2RSB order parameter for a diluted model because we assumed all the sites to be equivalent [14]. Notice in fact the finite neighborhood of any two sites $i$ and $j$ are identical (up to a gauge transformation $\sigma_{i} \rightarrow \tau_{i} \sigma_{i}$ ) in the thermodynamic limit.

We consider now a generic model such that, once an interaction term has been taken away, each adjacent spin interacts with $k$ other ones [to make contact with the Hamiltonian (6) one should take $k=l(p-1)]$. The 2RSB saddle point equations for such a model have the form

$$
Q[\rho]=\frac{1}{\mathcal{Z}} \int \prod_{i=1}^{k} d Q\left[\rho_{i}\right] z\left[\rho_{1}, \ldots, \rho_{k}\right]^{m_{1} / m_{2}} \delta\left[\rho-\rho_{0}\left[\rho_{1}, \ldots, \rho_{k}\right]\right],
$$

where $m_{1}<m_{2}$ are the two breaking parameters required for 2RSB [18]. The functional $\rho_{0}[\ldots]$ reads

$$
\rho_{0}(x)=\frac{1}{z\left[\rho_{1}, \ldots, \rho_{k}\right]} \int \prod_{i=1}^{k} d \rho_{i}\left(x_{i}\right) w\left(x_{1}, \ldots, x_{k}\right)^{m_{2}} \delta\left(x-f\left(x_{1}, \ldots, x_{k}\right)\right),
$$

and $z\left[\rho_{1}, \ldots, \rho_{k}\right]$ is fixed by the normalization condition $\int d x \rho .(x)=1$. The functions $w\left(x_{1}, \ldots, x_{k}\right)$ and $f\left(x_{1}, \ldots, x_{k}\right)$ are model-dependent. Their explicit form for the model (6) are reported in App. A.

An 1RSB solution is recovered if $Q[\rho]$ is supported on $\delta$-functions. We want to compute the stability of this subspace under the recursion (8) . We consider therefore an order parameter $Q[\rho]$ which is concentrated on narrow distributions $\rho(x)$. Such distributions can be characterized by their first two moments:

$$
\bar{x} \equiv \int d \rho(x) x, \quad \epsilon \equiv \int d \rho(x) x^{2}-\left(\int d \rho(x) x\right)^{2} .
$$

In the $\epsilon \rightarrow 0$ limit we can derive from Eq. (8) a recursion for the probability distribution 
$Q(\bar{x}, \epsilon)$ of these two parameters:

$$
Q(\bar{x}, \epsilon) \approx \frac{1}{\hat{\mathcal{Z}}} \int \prod_{i=1}^{k} d Q\left(\bar{x}_{i}, \epsilon_{i}\right) w\left(\bar{x}_{1}, \ldots, \bar{x}_{k}\right)^{m_{1}} \delta\left(\bar{x}-f\left(\bar{x}_{1}, \ldots, \bar{x}_{k}\right)\right) \delta\left(\epsilon-\sum_{i=1}^{k}\left(\partial_{\bar{x}_{i}} f\right)^{2} \epsilon_{i}\right)
$$

Notice that the largest breaking parameter $m_{2}$ plays no role in the relation (11). Hereafter we shall drop the subscript in the remaining parameter and set $m=m_{1}$. Let us call $\rho_{*}(\bar{x})$ the marginal distribution of $\bar{x}: \rho_{*}(\bar{x}) \equiv \int d \epsilon Q(\bar{x}, \epsilon)$. It is straightforward to see that this distribution satisfies the 1RSB equation:

$$
\rho_{*}(\bar{x})=\frac{1}{z_{*}} \int \prod_{i=1}^{k} d \rho_{*}\left(\bar{x}_{i}\right) w\left(\bar{x}_{1}, \ldots, \bar{x}_{k}\right)^{m} \delta\left(\bar{x}-f\left(\bar{x}_{1}, \ldots, \bar{x}_{k}\right)\right) .
$$

Therefore $\rho_{*}(\bar{x})$ is nothing but the 1 RSB solution with parameter $m$. If $Q(\bar{x}, \epsilon) \rightarrow \rho_{*}(\bar{x}) \delta(\epsilon)$ under the iteration (11), the 1RSB space is stable. In the opposite case, it is unstable towards 2RSB and (presumably) FRSB.

The distribution $Q(\bar{x}, \epsilon)$ can be represented by a population of couples $\left\{\left(\bar{x}_{i}, \epsilon_{i}\right): i=\right.$ $1, \ldots, \mathcal{N}\}$, and the recursion (111) can be approximated by a population dynamics algorithm following the ideas of Ref. [17]. The stability of the 1RSB subspace can be verified by monitoring a suitable norm of the $\epsilon_{i}$ 's, e.g.

$$
\|\epsilon\| \equiv \frac{1}{\mathcal{N}} \sum_{i=1}^{\mathcal{N}}\left|\epsilon_{i}\right|
$$

and checking whether $\|\epsilon\| \rightarrow 0$ or not. An alternative (and numerically preferable) procedure is the following. After each sweep in the population dynamics algorithm the $\epsilon_{i}$ 's are renormalized: $\epsilon_{i} \leftarrow \epsilon_{i} / \lambda$. The parameter $\lambda$ is chosen such that $\|\epsilon\|=1$ is kept fixed (this can be done because Eq. (11) is linear in the $\epsilon_{i}$ 's). We keep track of $\lambda$ and (eventually) average it over many iterations. The 1RSB subspace is stable if $|\lambda|<1$.

In Tab. II we report the value $e_{\mathrm{G}}(T)$ of the internal energy on the marginally stable states $(|\lambda|=1)$ for the model ([6) with $p=3$ and several values of $l$. As for the the dynamic and static energies, $e_{\mathrm{d}}(T)$ and $e_{\mathrm{S}}(T)$, we used populations of size $\mathcal{N}=10^{5} \div 10^{6}$ and averaged over $200 \div 400$ iterations.

One further entry in Tab. II is the iso-complexity energy $e_{\mathrm{ic}}(T)$. The corresponding parameter $m_{\mathrm{ic}}(T)$ is defined by the condition

$$
\Sigma_{T}\left(m_{\mathrm{ic}}(T)\right)=\Sigma_{T_{\mathrm{d}}}\left(m_{d}\left(T_{d}\right)\right)
$$




\begin{tabular}{|l|l|}
$c=4$ & \begin{tabular}{l}
$e_{\mathrm{S}}(T)=-1.21771+0.06409 e^{-2 \beta}+0.5787 e^{-4 \beta}$ \\
$e_{\mathrm{d}}(T)=-1.15267-0.05816 T+0.08068 T^{2}+(-0.59409+0.65230 T) e^{-2 \beta}$ \\
$e_{\mathrm{G}}(T)=-1.16667-0.04109 T+0.10430 T^{2}+(-0.63535+0.47338 T) e^{-2 \beta}$ \\
$e_{\mathrm{ic}}(T)=-1.17565+0.14471 e^{-2 \beta}-0.85310 e^{-4 \beta}+33.618 e^{-6 \beta}$ \\
$e_{\mathrm{S}}(T)=-1.39492+0.0994375 e^{-2 \beta}+0.842632 e^{-4 \beta}$ \\
$e_{\mathrm{d}}(T)=-1.32540-0.03884 T+(1.79894-3.17138 T) e^{-2 \beta}+13.2849 e^{-4 \beta}$ \\
$e_{\mathrm{G}}(T)=-1.35728+0.011316 T+0.05804 T^{2}+(-0.43749+0.29550 T) e^{-2 \beta}$ \\
$e_{\mathrm{ic}}(T)=-1.35122+0.13262 e^{-2 \beta}+1.4556 e^{-4 \beta}$ \\
\hline$c=8$ \\
$e_{\mathrm{S}}(T)=-1.80920+0.07186 e^{-2 \beta}+0.10056 e^{-4 \beta}+2.9090 e^{-6 \beta}$ \\
$e_{\mathrm{d}}(T)=-1.70826-0.04277 T+(1.0528+0.3575 T) e^{-4 \beta}$ \\
$e_{\mathrm{G}}(T)=-1.77828-0.00159 T+0.07624 T^{2}+(-2.7278+1.3729 T) e^{-4 \beta}$ \\
$e_{\mathrm{ic}}(T)=-1.76036+(1.0636+0.2779 T) e^{-4 \beta}$
\end{tabular} \\
\hline
\end{tabular}

TABLE II: The static $\left(e_{\mathrm{s}}\right)$, dynamic $\left(e_{\mathrm{d}}\right)$, marginal $\left(e_{\mathrm{G}}\right)$ and iso-complexity $\left(e_{\mathrm{ic}}\right)$ energies as obtained through a population dynamics solution of the 1RSB equations. We present here a compact representation of these curves as polynomials in $T$ and $e^{-2 \beta}$. The uncertainty due to statistical fluctuations of the population dynamics algorithm is $\Delta e \lesssim 0.0005$ for $c=4,5$ (and $\Delta e \lesssim 0.001$ for $c=8)$. For $e_{\mathrm{ic}}(T)$ the systematic error due to the uncertainty on $\Sigma_{\mathrm{d}}\left(T_{\mathrm{d}}\right)$ has to be added.

where, with an abuse of notation, we used the symbol $\Sigma_{T}(\cdot)$ to denote the complexity as a function of the breaking parameter. The relevance of this curve to the issues introduced in Sec. [1 was suggested in Ref. [19] and will be discussed in the next Section.

\section{NUMERICAL RESULTS}

We simulated the model (6) for $p=3$ and $c=4,5,8$. In order to investigate the question in Eq. (3), we measured the internal energy of the system, $\mathcal{O}=\mathcal{H}(\sigma)$, both during the relaxation after a quench and during a slow cooling.

The system is always prepared in a random configuration $(T=\infty)$. In the quenching experiments the system is let evolve directly at the final temperature $T_{\mathrm{f}}$. In the cooling experiments we used the following schedule for the temperature: $n_{\text {cool }}$ Monte Carlo sweeps (MCS) are performed at each temperature $T \in\left\{T_{\max }, T_{\max }-\Delta T, T_{\max }-2 \Delta T, \ldots, 2 \Delta T, \Delta T, 0\right\}$. Val- 


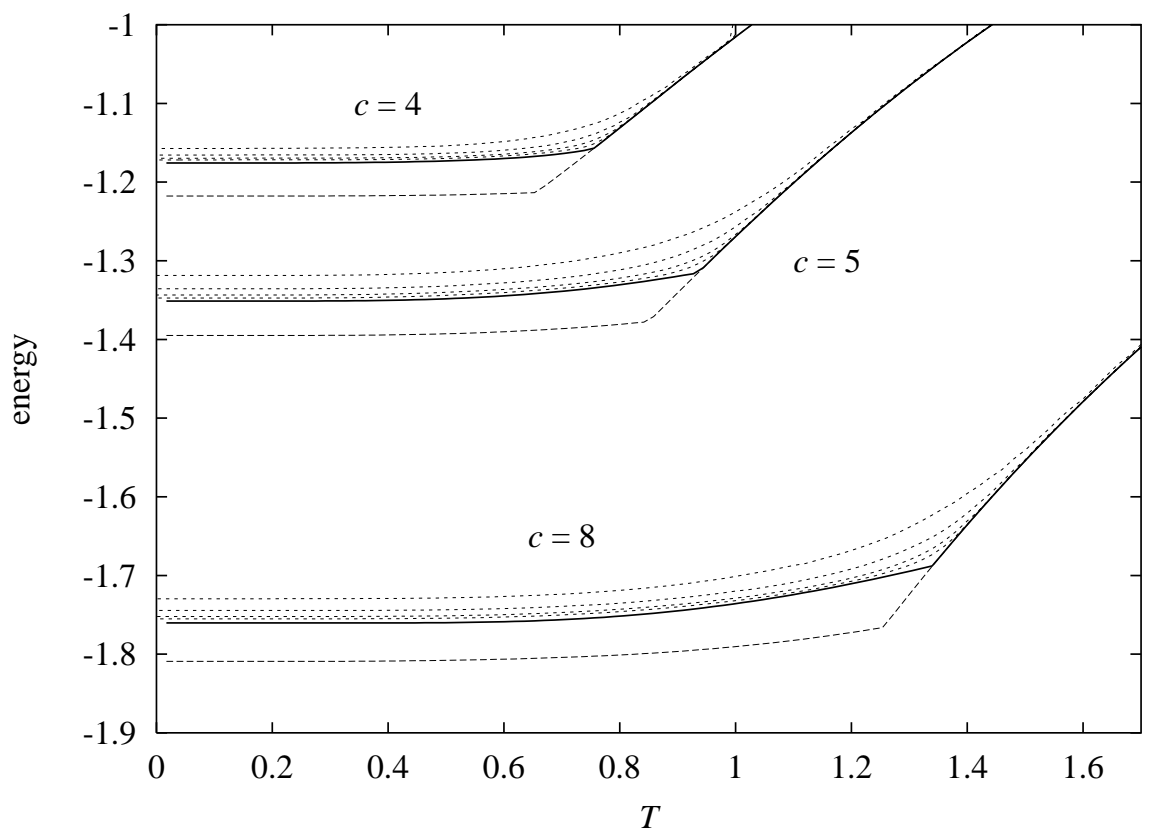

FIG. 2: Cooling experiments for several values of the connectivity $c$ and of the cooling rate $n_{\text {cool }}$. Dotted lines represent the numerical results for $e_{\mathrm{cool}}\left(T, n_{\mathrm{cool}}\right)$. Dashed lines correspond to the analytical result for the equilibrium energy, and solid lines to the paramagnetic energy (for $T>T_{\mathrm{d}}$ ) or to the iso-complexity energy $e_{\mathrm{ic}}(T)$ (for $\left.T<T_{\mathrm{d}}\right)$.

ues for $n_{\text {cool }}$ are $10^{2}, 10^{3}, 10^{4}$ and $10^{5}$, while those for $T_{\max }$ and $\Delta T$ are reported in the two central columns of Tab. I.

In order to minimize finite size effects, we considered samples of size $N=\left(4 \times 10^{5}-1\right)$ for quenching experiments, and $N=\left(10^{5}-1\right)$ for cooling experiments. We checked our results simulating few samples of size $N=\left(10^{6}-1\right)$. The number of samples varies from $N_{\mathrm{s}}=5$ for quenching experiments to $N_{\mathrm{s}} \gtrsim 10$ for cooling experiments.

In Fig. 2 we show the internal energy of the system as a function of the temperature during the cooling experiments with 4 different values of $n_{\text {cool }}$ for each connectivity (upper dotted lines). It should be clear from this picture that the system is undergoing a dynamic arrest preventing it to reach the thermodynamic energy (lower dashed line). We also show in this picture the iso-complexity energy (bold solid line). Numerical evidences, to be shown below, strongly suggests that the system follows the iso-complexity line when cooled infinitely slowly.

In order to better study the $n_{\text {cool }} \rightarrow \infty$ limit we choose one connectivity, e.g. $c=5$, and 


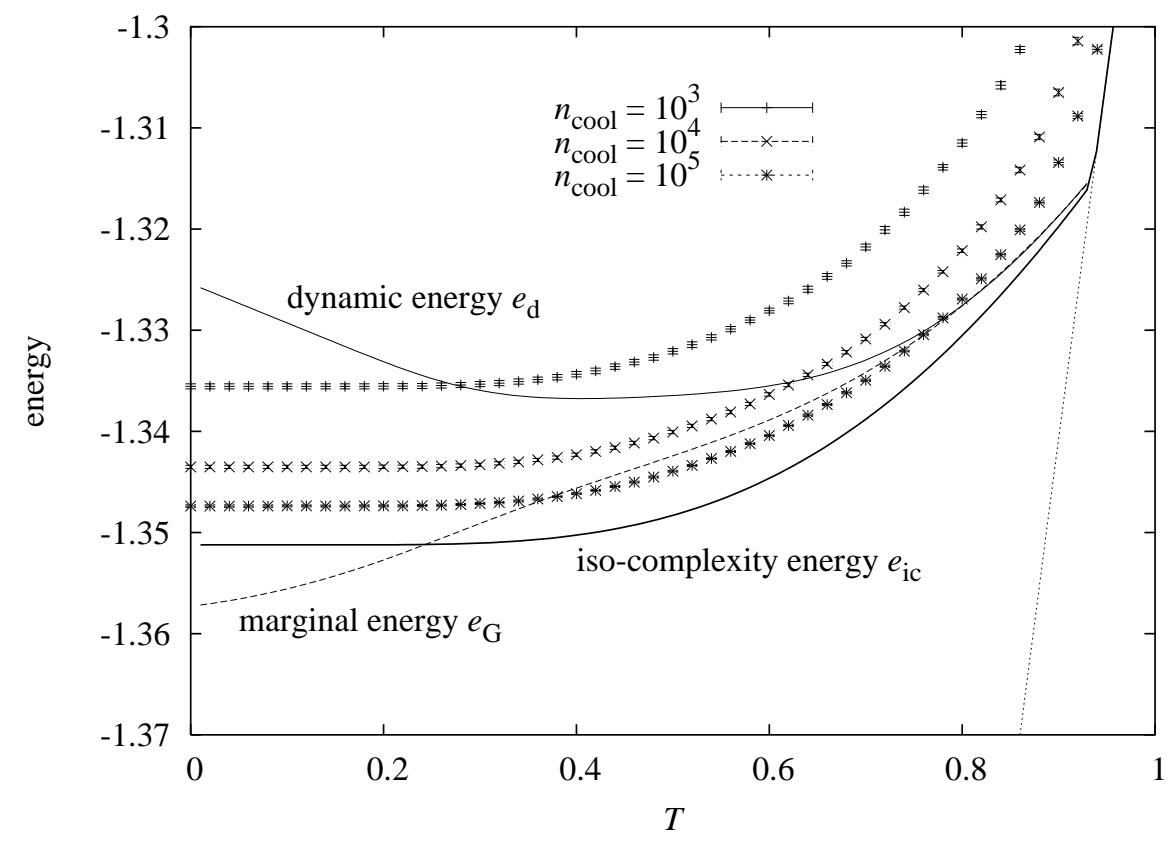

FIG. 3: A closer look at the cooling experiments for connectivity $c=5$.

we zoom on the interesting region. Figure 3 shows cooling energies for $c=5$ on a different scale $\left(n_{\text {cool }}=10^{2}\right.$ has been omitted for clarity). Statistical errors are smaller than symbol size. For comparison we also plot (from top to bottom) the analytic curves corresponding to the threshold or dynamic energy, $e_{\mathrm{d}}(T)$, the marginal energy, $e_{\mathrm{G}}(T)$, and the iso-complexity energy, $e_{\mathrm{ic}}(T)$, reported in Tab. II]

It is expected that energy relaxation converges to a threshold energy, lying between $e_{\mathrm{G}}(T)$ and $e_{\mathrm{d}}(T)$. This belief is based on the fact that for energies below $e_{\mathrm{G}}(T) 1 \mathrm{RSB}$ states are stable. On the contrary we clearly see from Fig. 3 that a cooling experiments may bring the system to an energy below $e_{\mathrm{G}}(T)$. In Sec. IV we will argue that this numerical observation is not in contrast with the existence of well defined 1RSB states.

Next we ask whether for very slow cooling rates, i.e. for $n_{\text {cool }} \rightarrow \infty$, the energy of the system follows the iso-complexity energy. In order to investigate this point we extrapolate the cooling energy for $n_{\text {cool }} \rightarrow \infty$ as follows. For any temperature $T$ we fit the data to the law

$$
e_{\mathrm{cool}}\left(T, n_{\mathrm{cool}}\right)=e_{\mathrm{cool}}(T)+a n_{\mathrm{cool}}^{-b}
$$

For all the connectivities considered $(c=4,5,8)$ and for temperatures $T \lesssim T_{\mathrm{d}}$ typical values 


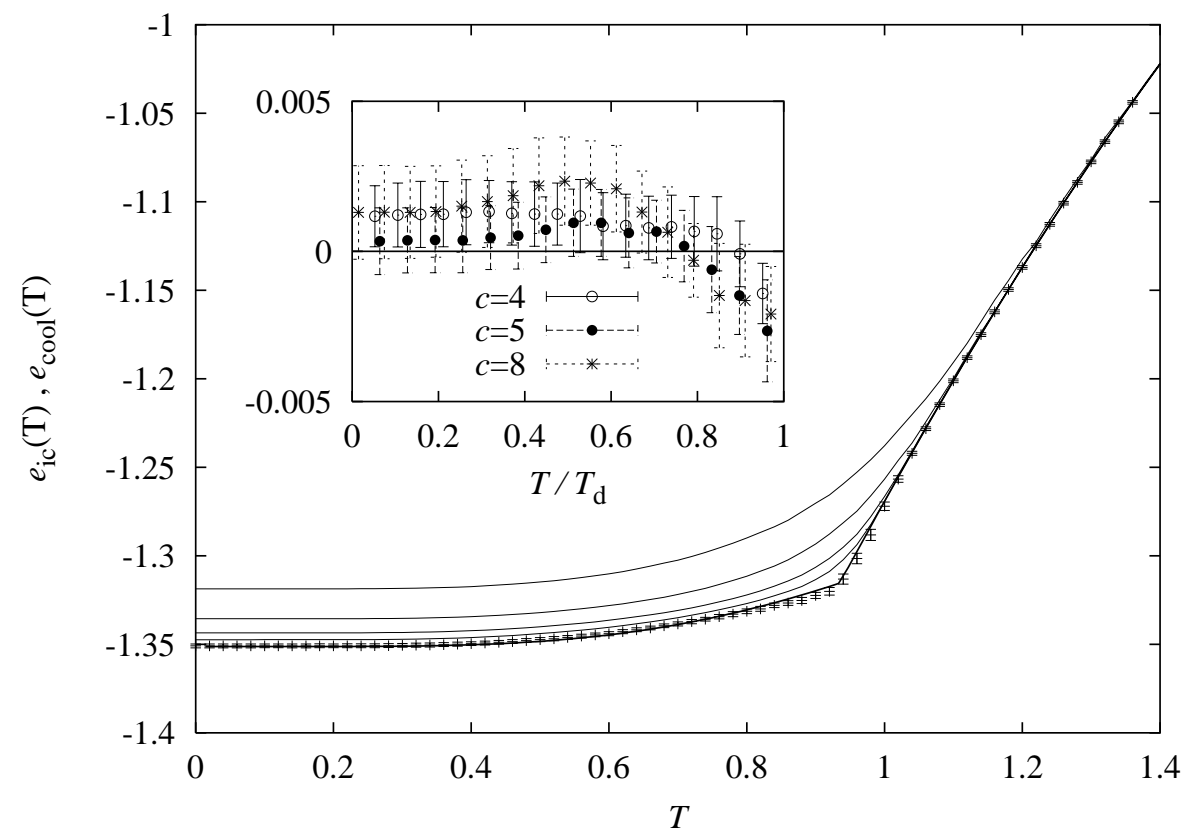

FIG. 4: Extrapolation of the cooling experiments to infinitely slow cooling rate. The crosses with error bars in the main frame represent the extrapolated data. Error bars include the uncertainty in the analytical calculation of $e_{\mathrm{ic}}(T)$. In the inset: difference between the extrapolated data and the analytical result for $e_{\text {ic }}(T)$, for connectivities $c=4,5,8$.

for the best fitting $b$ parameter are in the range $0.3 \div 0.36$.

The extrapolated energies $e_{\text {cool }}(T)$ for $c=5$ are plotted in the main panel of Fig. 4 (points with errors). We have included in the error bars also the contribution due to the uncertainty on the analytic estimation of $e_{\mathrm{ic}}(T)$. Thin lines are cooling energies with $n_{\text {cool }}=$ $10^{2}, 10^{3}, 10^{4}, 10^{5}$, while the thick line is the iso-complexity energy.

Within the statistical error $e_{\text {cool }}(T)$ and $e_{\text {ic }}(T)$ perfectly coincide, as can been better seen in the inset of Fig. 4 , where the difference $e_{\text {cool }}(T)-e_{\mathrm{ic}}(T)$ has been plotted for all the connectivities.

Let us now consider the energy relaxation after a sudden quench to temperature $T$. The temperatures studied for each connectivity are written in the last column of Tab. I Each simulations has been run for $10^{6}$ MCS. We assume that on late times the energy relaxation may be approximated by a single power law behavior

$$
e_{\text {quench }}(T, t)=e_{\text {quench }}(T)+a^{\prime} t^{-b^{\prime}} \text {. }
$$




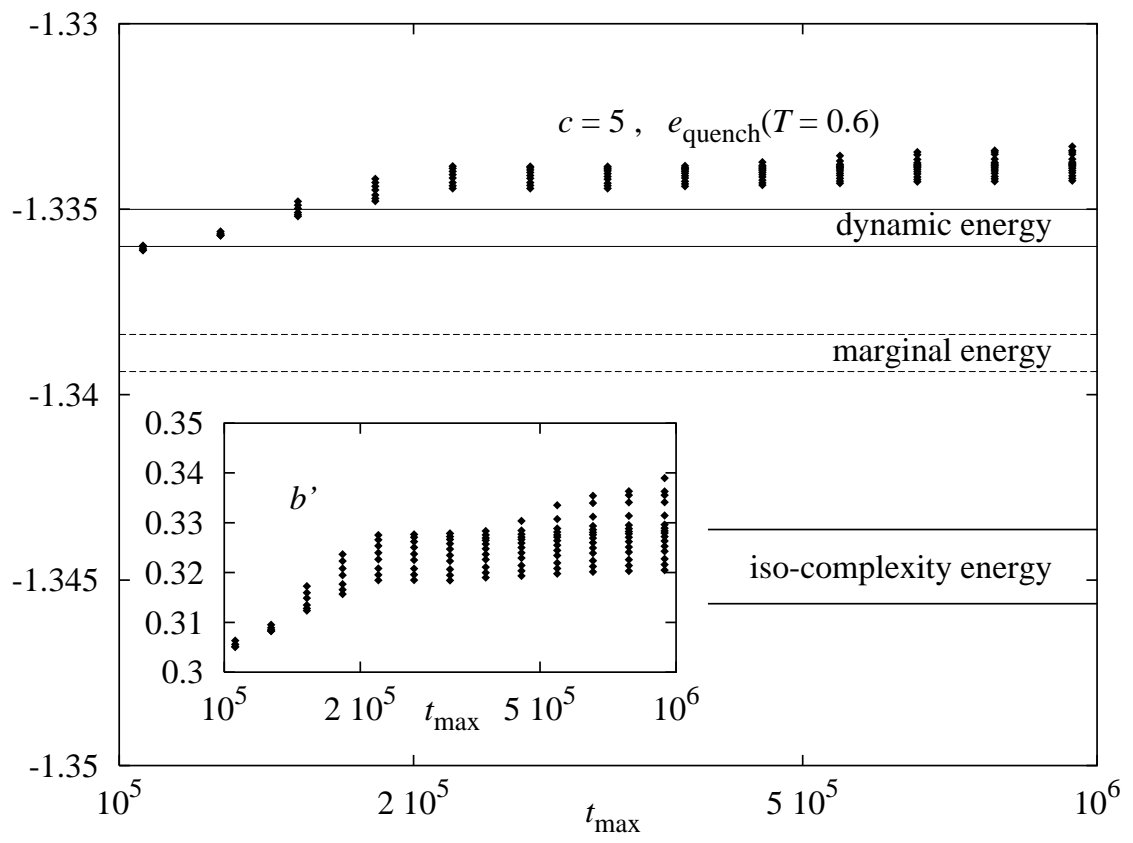

FIG. 5: The energy extrapolated from the quenches (main panel) and the best fitting $b^{\prime}$ parameter (inset), for $c=5$ and $T=0.6$. Each analytical energy is shown with 2 horizontal lines corresponding to its mean plus or minus one standard deviation.

Best fitting values for $b^{\prime}$ are typically around $1 / 3$, for all the connectivities and the temperatures studied.

The extrapolation of $e_{\text {quench }}(T)$ depends of course on the range of times used in the fitting procedure, because of sub-leading corrections. In order to have an appreciation of this effect, we fitted the $e_{\text {quench }}(T, t)$ data to expression (15) within the interval $t \in\left[t_{\min }, t_{\max }\right]$, with $t_{\max } \geq 10^{5}$ and $t_{\max } / t_{\min } \geq 200$.

In Fig. [5 the extrapolated energy $e_{\text {quench }}(T)$ (in the main panel) and the best fitting $b^{\prime}$ (in the inset) are shown as a function of $t_{\max }$ for $c=5$ and $T=0.6$ (very similar results has been obtained for the remaining connectivities and temperatures). For each value of $t_{\max }$, different points correspond to different values of $t_{\min }$. Statistical errors (not shown for clarity) are of the same order of the spread of points. Results seems to be almost $t_{\min }$-independent, and also the dependence on $t_{\max }$ is rather mild.

From Fig. [ we conclude that the extrapolated energy value is reasonably robust. Moreover the comparison with analytic energies supports the conclusion that $e_{\text {quench }}(T)$ stays above the energy $e_{\mathrm{ic}}(T)$ reached by cooling experiments. 
On the other hand we expect $e_{\text {quench }}(T)$ to coincide with the FRSB dynamic energy $e_{\mathrm{d}, \mathrm{FRSB}}(T)$, i.e. with the maximal energy such that the complexity is strictly positive, once FRSB effects have been taken into account [12]. While we know that $e_{\mathrm{G}}(T) \leq e_{\mathrm{d}, \mathrm{FRSB}}(T) \leq$ $e_{\mathrm{d}}(T)$, we do not yet have any estimate of this threshold. Verifying this theoretical expectation is therefore very difficult. Based on the data of Fig. 5 we guess that, if our expectation proves correct, then $e_{\mathrm{d}, \mathrm{FRSB}}(T)$ is 'close' to $e_{\mathrm{d}}(T)$ in the models at hand. The vicinity of the energy extrapolated from the quenches $e_{\text {quench }}(T)$ to the dynamic energy $e_{\mathrm{d}}(T)$ is well verified for all the connectivities and the temperatures studied in this work (see last column of Tab. I). This is presumably a general property of the model (6) for the range of connectivities considered in this paper.

\section{DISCUSSION}

We think that previous pages provide solid support for three statements concerning the general question (3): $(i) e_{\text {cool }}(T)<e_{\text {quench }}(T) ;(i i) e_{\text {quench }}(T)>e_{\mathrm{G}}(T) ;(i i i) e_{\text {cool }}(T)=e_{\mathrm{ic}}(T)$. For certain values of the temperature also a fourth statement holds: $(i v) e_{\text {cool }}(T)<e_{\mathrm{G}}(T)$. The first of these statements, in particular, implies a negative answer to the question (3). While the second statement was largely expected [12], the fourth one comes as a surprise, being in contradiction with some widespread expectation in the field. Notice moreover that these statements gets sharper as the temperature is lowered. In fact the separation between $e_{\mathrm{d}}(T), e_{\mathrm{ic}}(T)$ and $e_{\mathrm{G}}(T)$ increases at low temperature, cf. Fig. [3.

As for the third one, namely $e_{\text {cool }}(T)=e_{\text {ic }}(T)$, there exists a simple argument which helps to understand this relation. Consider a system with 1RSB, and assume that no temperature chaos is present. In other words, pure states can be traced in temperature without crossings

or bifurcations. It is then easy to show [19] that the energy of a pure state, regarded as a function of the temperature, follows an iso-complexity line (the value of the complexity on this line depending upon the state). In particular, $e_{\mathrm{ic}}(T)$ is the energy of the first metastable states to appear when the temperature is lowered across $T_{\mathrm{d}}$. Therefore, as far as single-time quantities are concerned, the system behave as if it equilibrated within the first metastable states encountered in its cooling history. Of course this picture is too simplified, and in fact the system ages also if it is slowly cooled across $T_{\mathrm{d}}$ [20]. A pictorial view of the evolution of the system during a slow cooling given in Fig. [1] 
Coming back to the discussion of metastable states in Sec. [1. the present analysis suggest a general (and testable) strategy for tailoring out-of-equilibrium ensembles of the form (5). The general idea is to add external sources $\lambda_{1}, \lambda_{2}$, etc conjugated to extensive observables $\mathcal{O}_{1}, \mathcal{O}_{2}$, etc. Such observables could be, for instance, the energy or the magnetization. The important rule is that any slow change in the control parameters induces an iso-complexity change in the state of the system. This is analogous to entropy conservation for adiabatic transformations in classical thermodynamics. Although here the system is in contact with a thermal bath, slow degrees of freedom are effectively thermally isolated.

Let us finally comment on the relevance of our results for the analysis of local search algorithms for random combinatorial optimization problems (here we make the usual identification between cost function and Hamiltonian). It has become customary [4, 21, 22] to compare the asymptotic cost $e_{\text {search }}(t \rightarrow \infty)$ achieved by such algorithms with the 1RSB threshold energy $e_{\mathrm{d}}(T=0)$. Here (see also [4] for a discussion concerning this point) we notice that:

1. In general, the local search algorithm does not satisfy detailed balance with respect to the Boltzmann distribution at any temperature $T$. If this is the case, the very existence of a relation between $e_{\text {search }}(t \rightarrow \infty)$ and any thermodynamic quantity [and a fortiori $\left.e_{\mathrm{d}}(T=0)\right]$ is an open problem.

2. In the simplest case, the local search algorithm satisfies detailed balance at any time $t$ with respect to the Boltzmann distribution at temperature $T(t)$, with $T(t)$ a deterministic schedule such that $T(t) \rightarrow 0$ as $t \rightarrow \infty$. Surprisingly, even in this case $e_{\mathrm{d}}(T=0)$ is not relevant. However, our results seem to imply that $e_{\text {search }}(t \rightarrow \infty) \geq e_{\mathrm{ic}}(T=0)$. This is an encouraging remark for the application of such algorithms. First of all $e_{\mathrm{ic}}(0)<e_{\mathrm{d}}(0)$. Moreover one could imagine constructing more complex annealing paths using clever deformations of the cost function to define the Hamiltonian. A smart deformation would probably allow to reduce $e_{\mathrm{ic}}(0)$.

\section{Acknowledgments}

We are grateful to Andrei Lopatin for bringing to our attention the relevance of the iso-complexity curve, and to Giorgio Parisi for stressing the importance of assuming the 
absence of temperature chaos. This work was partially supported by the ESF programme SPHINX and by the European Community's Human Potential Programme under contracts HPRN-CT-2002-00307, Dyglagemem, and HPRN-CT-2002-00319, Stipco.

\section{APPENDIX A: SOME FORMULAE FOR THE DILUTED $p$-SPIN MODEL}

For the sake of self-containedness we report here some explicit formulae for the diluted $p$-spin model (6). These formulae can be used in computing the 1RSB stability threshold along the lines of Sec. [1]

It is convenient to express the cavity equations in terms of two functional order parameter

$Q[\rho]$ and $\widehat{Q}[\widehat{\rho}]$. The first one is related to the distribution of cavity fields when one interaction term is removed from the system. The second one corresponds to the distribution of cavity fields when one spin is removed from the system.

The 2RSB cavity equations read

$$
\begin{aligned}
& Q[\rho]=\frac{1}{\mathcal{Z}} \int \prod_{i=1}^{l} d \widehat{Q}\left[\widehat{\rho}_{i}\right] z\left[\widehat{\rho}_{1}, \ldots, \widehat{\rho}_{l}\right]^{m_{1} / m_{2}} \delta\left[\rho-\rho_{0}\left[\widehat{\rho}_{1}, \ldots, \widehat{\rho}_{l}\right]\right], \\
& \widehat{Q}[\widehat{\rho}]=\int \prod_{j=1}^{p-1} d Q\left[\rho_{j}\right] \mathbb{E}_{J} \delta\left[\widehat{\rho}-\widehat{\rho}_{0}\left[J ; \rho_{1}, \ldots, \rho_{p-1}\right]\right],
\end{aligned}
$$

where $\mathbb{E}_{J}$ denotes the expectation with respect to the random variable $J$ which takes values +1 or -1 with equal probability). The mappings $\rho_{0}[\ldots]$ and $\widehat{\rho}_{0}[\ldots]$ are defined below:

$$
\begin{aligned}
& \rho_{0}(x)=\frac{1}{z\left[\left\{\widehat{\rho}_{i}\right\}\right]} \int \prod_{i=1}^{l} d \widehat{\rho}_{i}\left(y_{i}\right) w\left(\left\{y_{i}\right\}\right)^{m_{2}} \delta\left(x-y_{1}-\cdots-y_{l}\right), \\
& \widehat{\rho}_{0}(y)=\int \prod_{j=1}^{p-1} d \rho_{j}\left(x_{j}\right) \delta\left(y-\frac{1}{\beta} \operatorname{atanh}\left[\tanh \beta J \tanh \beta x_{1} \ldots \tanh \beta x_{p-1}\right]\right) .
\end{aligned}
$$

The reweighting factor is

$$
w\left(y_{1}, \ldots, y_{l}\right)=\frac{2 \cosh \left(\beta \sum_{i=1}^{l} y_{i}\right)}{\prod_{i=1}^{l} 2 \cosh \beta y_{i}}
$$

\section{APPENDIX B: THE LARGE CONNECTIVITY LIMIT}

The recipe we proposed for computing the stability condition of a diluted spin glass model does not necessarily capture the most relevant instability. In this Appendix we show that, 
in the large connectivity limit $l \rightarrow \infty$, our approach yields the replicon instability already computed in 13]. This provides an important check of our calculation.

When adapting the basic recursion (11) to the cavity equations reported in the previous Appendix, it is necessary to use two distributions $Q(\bar{x}, \epsilon)$ and $\widehat{Q}(\bar{y}, \hat{\epsilon})$. These distributions can be used to define the functions $\epsilon(x)$ and $\hat{\epsilon}(y)$ as follows

$$
\epsilon(x)=\int d \epsilon^{\prime} Q\left(x, \epsilon^{\prime}\right) \epsilon^{\prime}, \quad \hat{\epsilon}(y)=\int d \hat{\epsilon}^{\prime} \widehat{Q}\left(y, \hat{\epsilon}^{\prime}\right) \hat{\epsilon}^{\prime}
$$

It is easy to show that Eq. (11) implies a recursion of the type $[\epsilon(x), \hat{\epsilon}(y)] \mapsto\left[\epsilon^{\prime}(x), \hat{\epsilon}^{\prime}(y)\right]$, where:

$$
\epsilon^{\prime}(x)=\int d y M(x, y) \hat{\epsilon}(y), \quad \hat{\epsilon}^{\prime}(y)=\int d x \hat{M}(y, x) \epsilon(y) .
$$

The kernels of this mapping are given in terms of the $1 \mathrm{RSB}$ solution $\rho_{*}(x), \widehat{\rho}_{*}(y)$ :

$$
\begin{aligned}
& M(x, y)=\frac{l}{z_{*}} \int \prod_{i=1}^{l-1} d \widehat{\rho}_{*}\left(y_{i}\right) w\left(y, y_{1} \ldots y_{l-1}\right)^{m} \delta\left(x-y-y_{1}-\cdots-y_{l-2}\right), \\
& \hat{M}(y, x)=\int \prod_{j=1}^{p-2} d \rho_{*}\left(x_{j}\right) \Delta\left(x,\left\{x_{j}\right\}\right) \delta\left(y-\frac{1}{\beta} \operatorname{atanh}\left[\tanh \beta J \tanh \beta x \cdots \tanh \beta x_{p-2}\right]\right),
\end{aligned}
$$

where $z_{*}=z\left[\widehat{\rho}_{*} \ldots \widehat{\rho}_{*}\right]$ and

$$
\Delta\left(x,\left\{x_{j}\right\}\right) \equiv(p-1)\left[\frac{\tanh \beta J\left(1-\tanh ^{2} \beta x\right) \prod_{j=1}^{p-2} \tanh \beta x_{j}}{1-\tanh ^{2} \beta J \tanh ^{2} \beta x \prod_{j=1}^{p-2} \tanh ^{2} \beta x_{j}}\right]^{2} .
$$

Notice that we do not need to average on the sign of $J$ in Eqs. (B4) and (B5) because the distribution $\rho_{*}(x)$ is symmetric.

The stability of the fixed point $\epsilon(x)=0, \hat{\epsilon}(y)=0$ under the recursion (B2), can be determined by diagonalizing the composition of the kernels (B3), (B4). As we will see shortly, this diagonalization becomes considerably easier in the limit $l \rightarrow \infty$.

First of all, we rescale the coupling strength by setting $J=\sqrt{p / 2 l}$ in such a way that the energy remains finite in the large connectivity limit. We also rescale the cavity fields defining $y=\sqrt{p / 2 l} \tilde{y}$ (hereafter we shall drop the tilde). It is easy to see that, in the large connectivity limit, the 1RSB fields distributions become

$$
\begin{aligned}
& \rho_{*}(x)=\frac{1}{\mathcal{Z}}(\cosh \beta x)^{m} e^{-x^{2} / 2 \lambda} \\
& \widehat{\rho}_{*}(y)=\int \prod_{j=1}^{p-1} d \rho_{*}\left(x_{j}\right) \delta\left(y-\tanh \beta x_{1} \cdots \tanh \beta x_{p-1}\right) .
\end{aligned}
$$


The parameter $\lambda$ must be found by solving the equation

$$
\lambda=\frac{p}{2}\left[\int d \rho_{*}(x) \tanh ^{2} \beta x\right]^{p-1} .
$$

This equation was already found in Ref. [13] while solving the fully connected $p$-spin model.

Taking the $l \rightarrow \infty$ limit also in the kernels (B33) and (Bי1) we obtain

$$
\begin{aligned}
& M(x, y)=\frac{p}{2} \rho_{*}(x), \\
& \hat{M}(y, x)=\int \prod_{j=1}^{p-2} d \rho_{*}\left(x_{j}\right) D\left(x,\left\{x_{j}\right\}\right) \delta\left(y-\tanh \beta x \cdots \tanh \beta x_{p-2}\right),
\end{aligned}
$$

with

$$
D\left(x,\left\{x_{j}\right\}\right)=\left[\beta\left(1-\tanh ^{2} \beta x\right) \prod_{i=1}^{p-2} \tanh \beta x_{i}\right]^{2} .
$$

It is evident from Eq. (Bי) that there is a unique non-vanishing eigenvalue, and that the corresponding eigenvector has $\epsilon(x) \propto \rho_{*}(x)$. A little thought shows that the eigenvalue is

$$
\Lambda=\frac{1}{2} p(p-1) \beta^{2} q^{p-2} \int d \rho_{*}(x)(\cosh \beta x)^{-4},
$$

with $\rho_{*}(x)$ given by Eq. (B6 $)$. As anticipated, this result coincides with the replicon eigenvalue of [13].

[1] J.-P. Bouchaud, L. F. Cugliandolo, J. Kurchan and M. Mézard, in Spin Glasses and Random Fields, A. P. Young ed., (World Scientific, Singapore, 1997)

[2] T. R. Kirkpatrick and D. Thirumalai, Phys. Rev. B 36, 5388 (1987).

[3] O. Dubois, R. Monasson, B. Selman, and R. Zecchina. (eds.), Theor. Comp. Sci. 265, issue 1-2 (2001)

[4] S. Cocco, A. Montanari, R. Monasson, and G. Semerjian. Proceedings of Phase Transitions and Algorithmic Complexity 2002. E-print: cs.CC/0302003

[5] A. Crisanti and H.-J. Sommers, Z. Phys. B 87, 341 (1992).

[6] A. Crisanti, H. Horner, and H.-J. Sommers, Z. Phys. B 92, 257 (1993).

[7] L. F. Cugliandolo and J. Kurchan, Phys. Rev. Lett. 71, 173 (1993).

[8] M. Talagrand, Probab. Theor. Relat. Fields 117, 303 (2000) 
[9] R. Griffiths, C. Y. Weng, and J. S. Langer. Phys. Rev. 149, 301 (1966)

[10] S. Franz and G. Parisi, J. Physique 5, 1401 (1995)

[11] A. Barrat, S. Franz, and G. Parisi, J. Phys. A 30, 5593 (1997)

[12] A. Montanari and F. Ricci-Tersenghi, Eur. Phys. J B 33, 339 (2003)

[13] E. Gardner, Nucl. Phys. B257 [FS14], 747 (1985).

[14] S. Franz, M. Leone, F. Ricci-Tersenghi, and R. Zecchina, Phys. Rev. Lett. 87, 127209 (2001).

[15] S. Franz, M. Mézard, F. Ricci-Tersenghi, M. Weigt, R. Zecchina, Europhys. Lett. 55, 465 (2001)

[16] R. Monasson, Phys. Rev. Lett. 75, 2847 (1995).

[17] M. Mézard and G. Parisi, Eur. Phys. J. B 20, 217 (2001)

[18] M. Mezard, G. Parisi and M. A. Virasoro, Spin Glass Theory and Beyond (World Scientific, Singapore, 1987)

[19] A. V. Lopatin and L. B. Ioffe, Phys. Rev. B 66, 174202 (2002).

[20] F. Ricci-Tersenghi, unpublished.

[21] S. Franz, M. Leone, A. Montanari, and F. Ricci-Tersenghi, Phys. Rev. E 66, 046120 (2002).

[22] F. Ricci-Tersenghi, M. Weigt, and R. Zecchina, Phys. Rev. E 63, 026702 (2001).

[23] For the specialist: Throughout this paper we identify 'states' by the condition that two typical configuration in the same state have overlap greater or equal than $q_{\text {min }}$. Two configurations in different states have instead zero overlap. Here $q_{\min }$ is the smallest non-zero overlap in the support of the Parisi order parameter $P(q)$ (notice that such a definition is meaningful only for discontinuous models). Sometimes other names ('families', 'clusters', etc) are used for the same objects to emphasize that they can be decomposed further. 